\title{
$\begin{array}{ll}\text { Research Square } & \text { Preprints are preliminary reports that have not undergone peer review. } \\ \text { They should not be considered conclusive, used to inform clinical practice, }\end{array}$ or referenced by the media as validated information. \\ Fecundity disorders in older women: declines in follicular development and endometrial receptivity
}

\section{Li Wang}

The First Affiliated Hospital of Xi'an Jiaotong University, Xi'an, Shannxi,China

Shulan Lv

The First Affiliated Hospital of Xi'an Jiaotong University, Xi'an, Shaanxi, China

\section{Wenjun Mao}

The First Affiliated Hospital of Xi'an Jiaotong University, Xi'an, Shaanxi, China.

\section{E Bai}

The First Affiliated Hospital of Xi'an Jiaotong University, Xi'an, Shaanxi, China

\section{Xiaofeng Yang ( $\nabla$ yxf7_3@163.com )}

The First Affiliated Hospital of Xi'an Jiaotong University https://orcid.org/0000-0002-7631-4984

\section{Research article}

Keywords: Older women, Fecundity, Endometrial receptivity, Implantation window, Ultrasonic parameters

Posted Date: January 2nd, 2020

DOI: https://doi.org/10.21203/rs.2.19865/v1

License: (c) (1) This work is licensed under a Creative Commons Attribution 4.0 International License. Read Full License

Version of Record: A version of this preprint was published at BMC Women's Health on June 1st, 2020. See the published version at https://doi.org/10.1186/s12905-020-00979-7. 


\section{Abstract}

Background Fecundity declines in older women are associated with decreased ovarian function and oocyte quality, but little research is available on endometrial receptivity in older women. This study aimed to assess follicular development and endometrial receptivity, and to evaluate ultrasonic parameters in predicting endometrial receptivity during the implantation window in older women.

Methods For this prospective case-control study, 224 older women and 215 women under the age of 35 were recruited. The follicular development and endometrial thickness were monitored by transvaginal ultrasound before ovulation. During the implantation window, defined as 6 to 7 days after ovulation, the pulsatility index $(\mathrm{PI})$ and resistance index $(\mathrm{RI})$ of the uterine arteries and subendometrial region were calculated based on colour Doppler, and the endometrial volume, vascularization index ( $\mathrm{VI})$, flow index $(\mathrm{FI})$ and vascularization flow index (VFI) were also determined using the VOCAL imaging program with 3D mode power Doppler. The ultrasonic parameters were used to assess endometrial receptivity in older women.

Results The serum anti-Mullerian hormone (AMH) concentration and antral follicle count (AFC) were significantly lower in older women than in controls $(P<0.05)$. The average diameter of the dominant follicle was significantly smaller, and the subendometrial region RI was significantly higher in older women compared with controls $(\mathrm{P}<0.05)$. The normal ovulation rate was significantly lower in older women than in controls $(P<0.01)$. The subendometrial region RI was significantly higher, and the endometrial VI, $\mathrm{FI}$, and VFI were significantly lower in older women compared with controls $(P<0.05)$. The biochemical pregnancy rate, clinical pregnancy rate and ongoing pregnancy rate of older women were significantly lower than those of controls $(P<0.05)$. The best ultrasonic parameter for predicting endometrial receptivity during the implantation window in older women was $\mathrm{VI}$, followed by $\mathrm{FI}$.

Conclusions Older women present decreased serum AMH concentrations and AFC, defined as indicators of ovarian reserve function. Older women are characterized by decreased follicular development and endometrial receptivity, which may lead to fecundity disorders.

\section{Background}

In recent years, later-age childbearing has become a trend in China, particularly as more women have steadily entered the workforce. In addition, with the implementation of the two-child policy in China, the number of older women ( $\geq 35$ years) who desire pregnancy is gradually increasing. However, fecundity declines significantly beginning at approximately 32 years of age and decreases more rapidly after 37 years of age because of a decrease in egg quality and a gradual increase in the circulating level of follicle-stimulating hormone (FSH) [1]. Therefore, many older women will face fecundity disorders, including decreased egg quality, pregnancy rate and live birth rate, as well as increased embryonic aneuploidy and abortion rate [2]. 
It is well known that fecundity declines in older women are associated with decreased ovarian function and oocyte quality. However, little research is available on endometrial receptivity in older women. Endometrial receptivity plays a crucial role in the implantation of the embryo, and its impairment has been shown to be one of the factors contributing to infertility in women. Transvaginal ultrasound is the first-line method to observe follicular development and endometrial growth because of its non-invasive characteristics, and ultrasonic parameters, including endometrial thickness, classification and blood flow, have been confirmed to reflect endometrial receptivity [3-4]. This study aimed to assess ovarian reserve function, follicular development and endometrial receptivity in older women and to evaluate the predictive value for endometrial receptivity of different ultrasonic parameters during the implantation window.

\section{Materials And Methods}

\section{Study design and participants}

This prospective case-control study was conducted in the First Affiliated Hospital of Xi'an Jiaotong University from January 2017 to April 2019. A total of 224 older women ( $\geq 35$ years) and 215 women under the age of 35 who wanted to become pregnant were recruited. Women were included if they had regular menstrual cycles (21-35 days), bilateral fallopian tubal patency detected by hysterosalpingogram, and partners with normal semen according to WHO criteria [5]. Women were excluded if they had polycystic ovarian syndrome, thyroid disease, gynaecological surgery, pelvic inflammatory diseases or endometriosis. Socio-demographic information such as age, body mass index (BMI), number of gravidity and deliveries, and menstrual cycle were obtained by a questionnaire. Sex hormone and anti-Mullerian hormone (AMH) concentrations were detected in our hospital laboratory. The baseline antral follicle count (AFC) was determined, which was defined as the total number of antral follicles (follicles measuring 2$10 \mathrm{~mm}$ ) in both ovaries. All participants gave their informed consent for inclusion before they participated in the study. The protocol was approved by the First Affiliated Hospital of Xi'an Jiaotong University Institutional Review Board.

\section{Outcome measures}

The follicular development, endometrial thickness (ET), pulsatility index (PI) and resistance index (RI) of the uterine arteries and subendometrial region were monitored by transvaginal ultrasound every two days before ovulation. Timed intercourse was advised when the dominant follicle reached $18 \mathrm{~mm}$ in diameter. In addition, during the implantation window, defined as 6 to 7 days after ovulation, the endometrial volume, vascularization index (VI), flow index (FI) and vascularization flow index (VFI) were determined using the virtual organ computer-aided analysis (VOCAL) imaging program with 3-D mode power Doppler. All ultrasonic parameters were measured three times, and the average value was used for the final statistical analysis. Luteinized unruptured follicular syndrome (LUFS) was defined as persistent existence or enlargement of follicles after maturation, thickening of follicle walls, and a strength echo in the point or grid shape or a cystic solid echo with great tension inside the follicle. 
Pregnancy rates were observed during this menstrual cycle, including biochemical pregnancy (defined as human chorionic gonadotropin level $\geq 10 \mathrm{mlU} / \mathrm{mL}$ measured at 2 weeks after ovulation), clinical pregnancy (defined as the presence of embryo in the uterine cavity) and ongoing pregnancy (confirmed as the presence of a foetus with heart motion at 12 weeks of gestation) [6].

The primary outcome included follicular development and ultrasonic parameters of endometrial receptivity. The secondary outcomes were the pregnancy rates and the predictive value of different ultrasonic parameters for endometrial receptivity in older women.

\section{Statistical analysis}

The data in this study were statistically analyzed by SPSS version 16.0.The descriptive statistics for continuous variables were given as mean \pm SD and analyzed by Student's t-test. Differences in dichotomous outcomes were compared by chi-square test. The evaluation of endometrial receptivity of different parameters was done by receiver operating characteristic (ROC) curves. $P<0.05$ was considered statistically significant.

\section{Results}

The data in Table 1 suggest that the serum AMH concentration and AFC were significantly lower in older women than in controls $(P<0.05)$. No significant difference was found when comparing other basic clinical data between the two groups $(P>0.05)$.

Table 1 Clinical data between older women and controls.

\begin{tabular}{lccc}
\hline \multicolumn{1}{c}{ Characteristics } & Older women $(\mathrm{n}=224)$ & Controls $(\mathrm{n}=215)$ & $P$ value $^{a}$ \\
\hline BMI $\left(\mathrm{kg} / \mathrm{m}^{2}\right)$ & $21.46 \pm 5.45$ & $22.09 \pm 4.87$ & 0.658 \\
Number of gravidity & $2.17 \pm 0.86$ & $2.35 \pm 0.90$ & 0.437 \\
Number of delivery & $0.97 \pm 0.42$ & $0.41 \pm 0.16$ & 0.039 \\
Menstrual cycle (days) & $30.12 \pm 5.53$ & $31.45 \pm 6.02$ & 0.225 \\
Basic concentrations & & & \\
FSH $(\mathrm{mIU} / \mathrm{mL})$ & $7.80 \pm 2.76$ & $6.35 \pm 2.01$ & 0.367 \\
$\mathrm{LH}(\mathrm{mIU} / \mathrm{mL})$ & $5.53 \pm 1.12$ & $5.48 \pm 1.29$ & 0.216 \\
$\mathrm{PRL}(\mathrm{ng} / \mathrm{mL})$ & $10.14 \pm 3.37$ & $8.45 \pm 2.48$ & 0.103 \\
$\mathrm{E}_{2}(\mathrm{pmol} / \mathrm{L})$ & $91.35 \pm 25.63$ & $102.47 \pm 30.42$ & 0.098 \\
$\mathrm{P}(\mathrm{nmol} / \mathrm{L})$ & $1.09 \pm 0.76$ & $1.17 \pm 0.90$ & 0.342 \\
$\mathrm{~T}(\mathrm{nmol} / \mathrm{L})$ & $0.92 \pm 0.41$ & $0.86 \pm 0.44$ & 0.677 \\
$\mathrm{AMH}(\mathrm{ng} / \mathrm{mL})$ & $1.02 \pm 0.58$ & $2.65 \pm 0.99$ & 0.019 \\
$\mathrm{AFC}(\mathrm{number})$ & $8.53 \pm 2.21$ & $15.67 \pm 4.32$ & 0.022 \\
\hline
\end{tabular}


Data given as mean \pm SD. ${ }^{\text {a }}$ T-test.

FSH: follicle stimulating hormone, LH: luteinizing hormone, PRL: prolactin, $\mathrm{E}_{2}$ : estradiol, P: progesterone, T: testosterone.

Figure 1 indicates the follicular development and the PI/RI of the uterine arteries and subendometrial region before ovulation. The average diameter of the dominant follicle on days 14,16 , and 18 of the menstrual cycle were significantly smaller, and the subendometrial region RI on days $12,14,16$, and 18 of the menstrual cycle were significantly higher in older women than in controls $(P<0.05)$ (Fig. 1A and F). However, no significant difference was found when comparing endometrial thickness, average uterine PI and RI, and subendometrial region PI between the two groups ( $P>0.05$ ) (Fig. 1B-E).

Figure 2 reveals that the normal ovulation rate in older women was significantly lower compared with controls [69.64\% (156/224) vs. 82.79\% (178/215), $P<0.01]$, and the without dominant follicle rate in older women was significantly higher than that in controls [21.88\% $(49 / 224)$ vs. $9.77 \%$ (21/215), $P<0.01]$. However, no significant difference was found when comparing the LUFS rate between the two groups [8.48\% (22/224) vs. $7.44 \%(16 / 215)$, $P>0.05]$.

Table 2 shows the ultrasonic parameters of endometrial receptivity during the implantation window in women with normal ovulation between the two groups. The subendometrial region RI was significantly higher, and the endometrial VI, FI, and VFI were significantly lower in older women than in controls $(P<0.05)$. The data in Table 3 indicate that the biochemical pregnancy rate, clinical pregnancy rate and ongoing pregnancy rate of older women were significantly lower than those of controls $(P<0.05)$.

Table 2. Ultrasonic parameters of endometrial receptivity during implantation window in women with normal ovulation between the two groups. 


\begin{tabular}{lccc}
\hline \multicolumn{1}{c}{ Parameters } & Older women $(\mathrm{n}=156)$ & Controls $(\mathrm{n}=178)$ & $P$ value $^{a}$ \\
\hline Average uterine PI & $2.03 \pm 0.74$ & $2.08 \pm 0.81$ & 0.897 \\
Average uterine RI & $0.76 \pm 0.13$ & $0.75 \pm 0.15$ & 0.903 \\
Subendometrial region PI & $0.98 \pm 0.24$ & $1.00 \pm 0.29$ & 0.890 \\
Subendometrial region RI & $0.70 \pm 0.12$ & $0.62 \pm 0.11$ & 0.041 \\
Endometrial thickness $(\mathrm{mm})$ & $9.01 \pm 2.41$ & $9.67 \pm 2.68$ & 0.335 \\
Endometrial volume $\left(\mathrm{cm}^{3}\right)$ & $2.93 \pm 0.87$ & $3.24 \pm 1.02$ & 0.126 \\
Endometrial VI $(\%)$ & $1.72 \pm 0.92$ & $3.04 \pm 1.13$ & 0.019 \\
Endometrial FI (0-100) & $19.5 \pm 4.65$ & $32.3 \pm 6.32$ & 0.015 \\
Endometrial VFI (0-100) & $0.33 \pm 0.02$ & $0.97 \pm 0.06$ & 0.007 \\
\hline
\end{tabular}

\section{Data given as mean \pm SD.}

a T-test.

Figure 3 shows that the best ultrasonic parameter for predicting endometrial receptivity during the implantation window in older women was VI (AUC $=0.889$, sensitivity $92.6 \%$ and specificity $85.4 \%$ ), followed by FI (AUC=0.838, sensitivity $90.7 \%$ and specificity $82.1 \%$ ).

\section{Discussion}

Although there are several indicators to evaluate ovarian function, age is the primary determinant of reproductive potential. Data suggest that among populations that do not use contraception, fertility rates decrease with increasing age of women [7]. The cumulative pregnancy rate observed across up to 12 insemination cycles was $74 \%$ for women younger than 31 years, decreased to $62 \%$ for women aged $31-$ 35 years and decreased further to $54 \%$ for women older than 35 years [1]. Therefore, the pregnancy success rate of older women is significantly reduced in regard to both natural conception and assisted reproductive technology [8]. According to reports in the literature, pregnancy loss is closely related to age. Whereas $9.9 \%$ of women younger than 33 years who conceive during IVF with a fresh embryo transfer experience pregnancy loss after foetal heart activity is observed, the rate of miscarriage progressively increases to $13.7 \%$ for women aged 35-37 years and to $19.8 \%$ for women aged 38-40 years [9]. Our findings show that the biochemical pregnancy rate, clinical pregnancy rate and ongoing pregnancy rate in older women were significantly lower than those in controls.

Several indicators can be used to assess ovarian reserve function, including $\mathrm{FSH}$, inhibin $\mathrm{B}, \mathrm{AMH}$ and AFC. The data in this study revealed that $\mathrm{AMH}$ and AFC were superior to menstrual cycles and FSH in evaluating ovarian function in older women, which is consistent with the results reported in most previous research [10-11]. As a woman ages, her oocyte and follicular pool declines, so AFC is a good indicator of ovarian function in women. As the oocyte and follicular pool declines, granulosa cells secrete less. 
Although the ability of $\mathrm{AMH}$ to predict reproductive potential is controversial, it is an excellent predictor of oocyte yield among women with infertility undergoing controlled ovarian hyperstimulation for in vitro fertilization (IVF) [12]. In addition, data from our study showed that the average diameter of the dominant follicle in older women was significantly smaller, and the normal ovulation rate was significantly lower in older women, which is associated with the fecundity declines of older women.

Embryo implantation is a complicated process in which the blastocyst interacts with the receptive endometrium. In the normal reproductive cycle of humans and mammals, there is a very short period during which the endometrium is receptive for embryo implantation, which is defined as 6 to 7 days after ovulation in the normal menstrual cycle. In the early stage of embryo implantation, angiogenesis is active, and the expression of various angiogenesis-related factors is increased, which provides support for embryonic development and pregnancy. Therefore, the blood supply of the endometrium is of great significance to its receptivity. Our previous results showed that transvaginal two-dimensional ultrasound could evaluate endometrial receptivity by detecting endometrial thickness and blood flow [13].

At present, three-dimensional energy Doppler ultrasound, which can reflect the vascular configuration of organ tissues and the richness of blood flow, has been used to assess endometrial receptivity [14]. Studies have confirmed that the value of this technique in evaluating endometrial receptivity is better than that of two-dimensional ultrasound, and it has been reported to be used to evaluate endometrial receptivity for in vitro fertilization-embryo transfer (IVF-ET) [15]. The data in our study showed that the subendometrial region RI was significantly higher and that the endometrial $\mathrm{VI}, \mathrm{FI}$, and VFI during implantation window were significantly lower in older women than in controls, which might be related to the decreased endometrial receptivity. Furthermore, the data in this study show that the best ultrasonic parameter for predicting endometrial receptivity during the implantation window in older women was $\mathrm{Vl}$, followed by FI. Wang et al. showed that increased endometrial blood flow in IVF-ET infertile women during follicular maturation was beneficial to pregnancy [16]. Other studies have found that threedimensional energy Doppler ultrasonography can assess endometrial receptivity and predict pregnancy outcome by detecting follicular maturation day and embryo transfer day with intrauterine and subintimal blood flow [17].

This study has some limitations. Fecundity disorders in older women are associated with multiple factors, including follicular dysplasia, increased aneuploidy and decreased endometrial receptivity. In addition, as age increases, the risks of reproductive diseases that may adversely affect fertility, such as fibroids, tubal disease, and endometriosis, also increase. Furthermore, this was a prospective case-control study in a single centre, and the sample size was relatively small. Therefore, the repeatability of the results of this study needs to be confirmed by multi-centre surveys with large sample sizes.

\section{Conclusions}

Older women present decreased ovarian reserve function, for which the predictive value of AMH and AFC is more sensitive. Older women present decreased follicular development and endometrial receptivity, 
which might be related to fecundity disorders. The results of this study provide new ideas for the improvement of pregnancy rate and reproductive outcomes in older women.

\section{Abbreviations}

BMI: body mass index; AMH: anti-Mullerian hormone; AFC: antral follicle count; LUFS: luteinized unruptured follicular syndrome; FSH: follicle stimulating hormone; LH: luteinizing hormone; PRL: prolactin; $\mathrm{E}_{2}$ : estradiol; $\mathrm{P}$ : progesterone; $\mathrm{T}$ : testosterone; $\mathrm{PI}$ : pulsatility index; RI: resistance index; $\mathrm{ET}$ : endometrial thickness; EV: endometrial volume; VI: vascularization index; FI: Flow index; VFI: vascularization flow index.

\section{Declarations}

\section{Acknowledgements}

We would like to thank all the women who participated in this study.

\section{Authors' contributions}

Study design: LW and SL. Methodology: WJ. Investigation: EB. Writing-review and editing: XF. All authors read and approved the final manuscript.

\section{Funding}

This research was funded by Basic Research Program of Natural Science of Shaanxi Province, China (No. 2019JM-569), and by Institutional Foundation of The First Affiliated Hospital of Xi'an Jiaotong University (No. 2019ZYTS-03).

\section{Ethics approval and consent to participate}

The First Affiliated Hospital of Xi'an Jiaotong University Institutional Review Board and all participants gave their informed consent for inclusion before they participated in the study.

\section{Availability of data and materials}

Not applicable.

\section{Competing interests}

The authors declare that they have no competing interests.

\section{Author details}

All the authors are from Department of Obstetrics and Gynecology, The First Affiliated Hospital of Xi'an Jiaotong University, Xi'an, Shaanxi, People's Republic of China. 


\section{References}

1.American College of Obstetricians and Gynecologists Committee on Gynecologic Practice and Practice Committee. Female age-related fertility decline. Committee Opinion No. 589. Fertil Steril. 2014;101(3):633634.

2.Stephen EH, Chandra A, King RB. Supply of and demand for assisted reproductive technologies in the United States: clinic- and population based data, 1995-2010. Fertil Steril. 2016;105(2):451-458.

3.Liu H, Zhang J, Wang B, Kuang Y. Effect of endometrial thickness on ectopic

pregnancy in frozen embryo transfer cycles: an analysis including 17,244 pregnancy cycles. Fertil Steril. 2019 Nov 11. pii: S0015-0282(19)32316-7.

4.Masrour MJ, Yoonesi L, Aerabsheibani $\mathrm{H}$. The effect of endometrial thickness and endometrial blood flow on pregnancy outcome in intrauterine insemination cycles. J Family Med Prim Care. 2019; 8(9):28452849 .

5.Elnaggar A, Farag AH, Gaber M, Hafeez MA, Ali MS, Atef AM. AlphaVBeta3 Integrin expression within uterine endometrium in unexplained infertility: a prospective cohort study. BMC Womens Health.2017; 17(1):90.

6.Chen ZJ, Shi Y, Sun Y, Zhang B, Liang X, Cao Y, et al. Fresh versus frozen embryos for infertility in the polycystic ovary syndrome. N Engl J Med. 2016; 375:523-533

7.Pantazis A, Clark SJ. A parsimonious characterization of change in global age-specific and total fertility rates. PLoS One. 2018; 13(1):e0190574.

8.ElMokhallalati Y, van Eekelen R, Bhattacharya S, McLernon DJ. Treatment-independent live birth after invitro fertilisation: a retrospective cohort study of 2,133 women. Hum Reprod. 2019; 34(8):1470-1478.

9.Farr SL, Schieve LA, Jamieson DJ. Pregnancy loss among pregnancies conceived through assisted reproductive technology, United States, 1999-2002. Am J Epidemiol. 2007; 165:1380-1388.

10.Helden JV, Weiskirchen R. Age-independent anti-Müllerian hormone (AMH) standard deviation scores to estimate ovarian function. Eur J Obstet Gynecol Reprod Biol. 2017; 213:64-70.

11.Kim C, Slaughter JC, Wang ET, Appiah D, Schreiner P, Leader B, et al. Anti-Müllerian hormone, follicle stimulating hormone, antral follicle count, and risk of menopause within 5 years. Maturitas. 2017; 102:1825 .

12. Steiner AZ, Pritchard D, Stanczyk FZ, Kesner JS, Meadows JW, Herring AH, et al. Association between biomarkers of ovarian reserve and infertility among older women of reproductive age. JAMA. 2017; 318(14):1367-1376. 
13.Wang L,Wen XQ, Lv SL, Zhao J, Yang T, Yang XF. Comparison of endometrial receptivity of clomiphene citrate versus letrozole in women with polycystic ovary syndrome: a randomized controlled study. Gynecol Endocrinol. 2019; 35(10):862-865.

14.Mohsen IA, Elkattan E, Nabil H, Khattab S. Effect of Metformin treatment on endometrial vascular indices in anovulatory obese/overweight women with polycystic ovarian syndrome using threedimensional power Doppler ultrasonography. J Clinic Ultrasound. 2013; 41(5):275-282.

15.Manisha CM, Chowdhary J, Swarankar ML, Bharadwaj SL. Predictive value of subendometrialendometrial blood flow assessment by transvaginal 3D power doppler on the day of HCG on clinical outcome of IVF cycles. Int J Res Med Sci. 2015;3(11):3114-3118.

16.Wang L, Qiao J, Li R, Zhen X, Liu Z. Role of endometrial blood flow assessment with color Doppler energy in predicting pregnancy outcome of IVF-ET cycles. Reprod Biol Endocrinol. 2010; 18(8):122.

17.Choudhary M, Chowdhary J, Swarankar ML,et al. Predictive value of subendometrial-endometrial blood flow assessment by transvaginal 3D power doppler on the day of HCG on clinical outcome of IVF cycles. Int J Res Med Sci. 2015; 3(11):3114-3118.

\section{Figures}



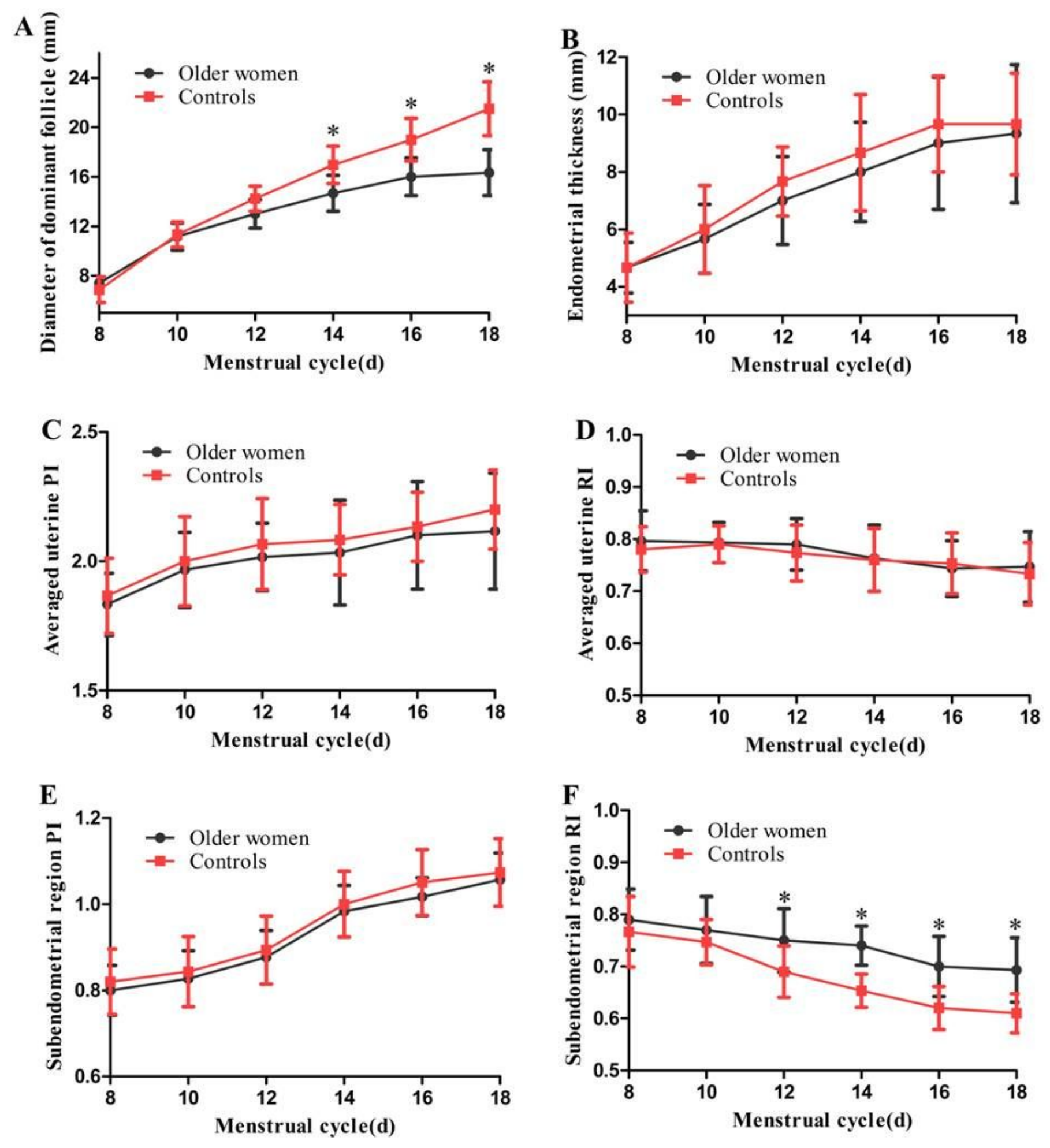

\section{Figure 1}

Follicular development, $\mathrm{PI} / \mathrm{RI}$ of the uterine arteries and subendometrial region before ovulation between older women and controls (PI: pulsatility index, RI: resistance index, $\left.{ }^{\mathrm{P}}<0.05\right)$. 


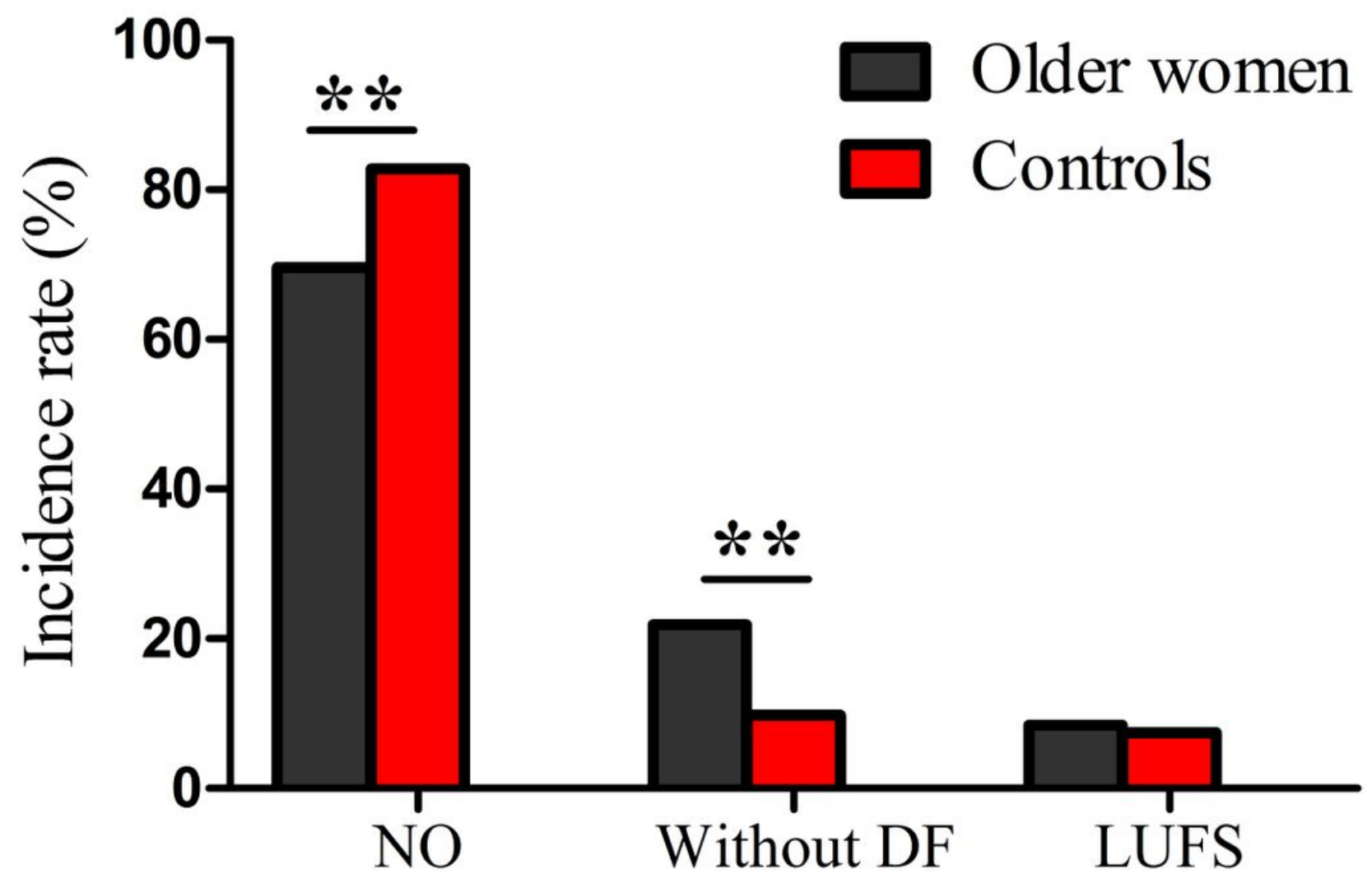

Figure 2

Follicular development and ovulation rate between older women and controls (NO: Normal ovulation, DF: Dominant follicle, LUFS: Luteinized unruptured follicular syndrome) 


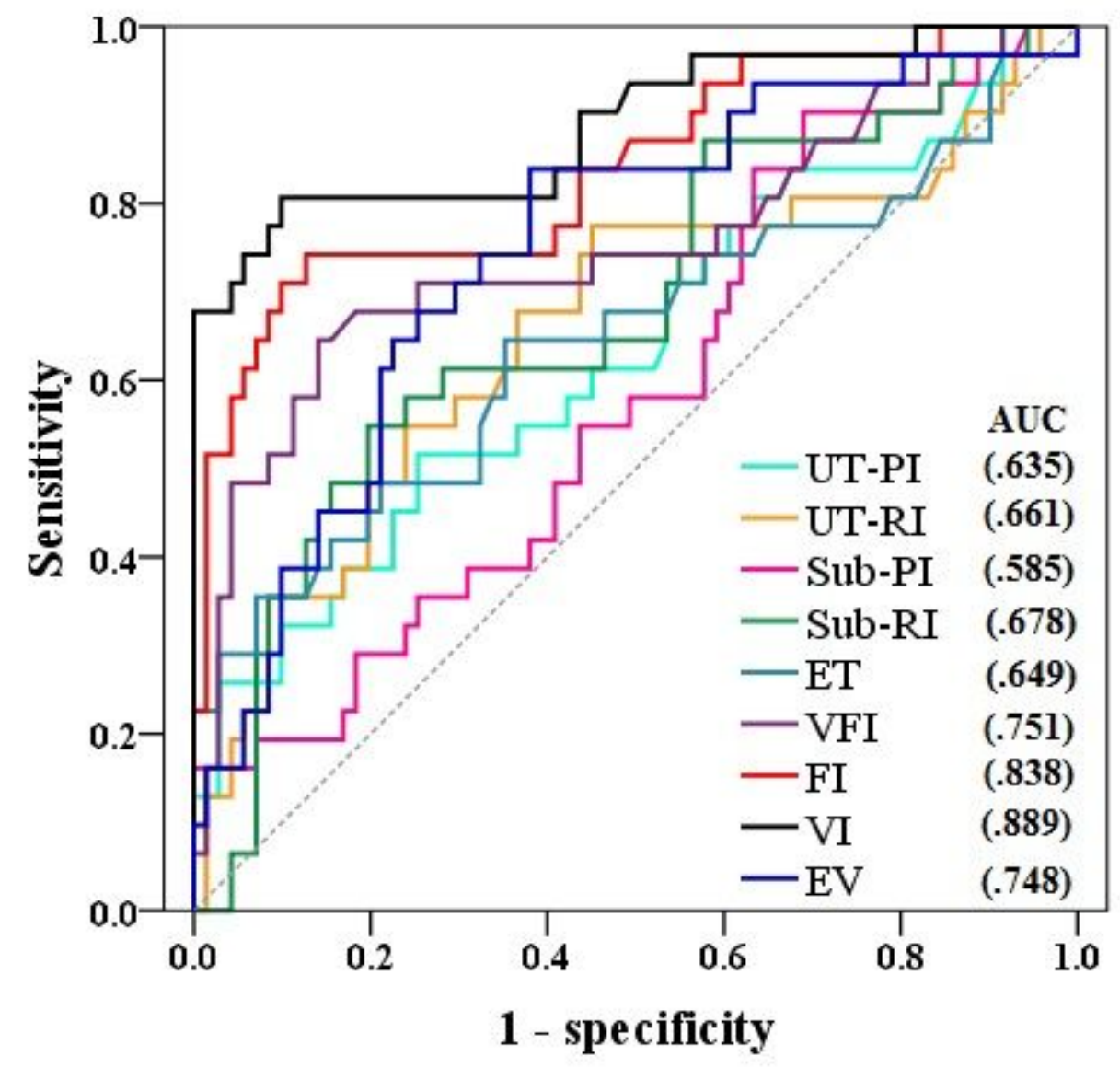

Figure 3

ROC curves of the predictive value for endometrial receptivity during implantation window in older women (UT-PI: uterine artery pulsatility index, UT-RI: uterine artery resistance index, Sub-PI: subendometrial region pulsatility index, Sub-RI: subendometrial region resistance index, ET: endometrial thickness, EV: endometrial volume, VI: vascularization index, Fl: Flow index, VFI: vascularization flow index). 